\section{CAMBIO DE PARADIGMA EN LOS ESTUDIOS DE INNOVACIÓN: EL GIRO SOCIAL DE LAS POLÍTICAS EUROPEAS DE INNOVACIÓN'}

\author{
Javier Echeverría Ezponda \\ Lucía Merino Malillos \\ Ikerbasque, Departamento de Sociología 2, UPV/EHU
}

\section{A CHANGE OF PARADIGM IN THE STUDY OF INNOVATION: THE SOCIAL TURN IN THE EUROPEAN POLICIES OF INNOVATION}

\begin{abstract}
Innovation studies have experienced a social shift in this first decade of the century, which begins to move to innovation policy. We are facing a new paradigm in such studies, not only to a further expansion of the types and models of innovation. To this end, we analyze some of the first conceptual frameworks of social innovation (Goldenberg and Crises group in Canada, Young Foundation and NESTA in the United Kingdom) and discussed the progress of the new paradigm in the European Union. We conclude that social innovation technosciences seem to have reached a broad consensus: social innovations emerge in the first instance of civil society, but can also be generated or implemented by the public sector and the private sector. In addition, both the means and the purposes to promote them should be predominantly social.
\end{abstract}

KEY WORDS: Social innovation; politics of innovation; social technosciences.

\section{Políticas de innovación de la Unión EuRopea en la Agenda de Lisboa 2000}

En una Comunicación de marzo de 2003 la Comisión Europea (CE) definió la política de innovación de la UE en el marco de la agenda de Lisboa². Tras constatar que los Indicadores de innovación 2002 mostraban que "los resultados de la Unión en el ámbito de la innovación siguen siendo escasos con relación a los de los Estados Unidos y de Japón" ${ }^{3}$, la CE alertaba de que el escaso crecimiento de la productividad en Europa era debido a una insuficiente innovación ${ }^{4}$. En consecuencia, señalaba el riesgo de que no se alcanzara el objetivo principal de la estrategia diseñada en Lisboa: conseguir el liderazgo mundial de la UE en la sociedad del conocimiento.

Dicho riesgo se vio confirmado tres años después, cuando la UE comprobó que el objetivo señalado en 2000 no sería
RESUMEN: Los estudios de innovación han experimentado un giro social en esta primera década del siglo XXI, que comienza a trasladarse a las políticas de innovación. Estamos ante un nuevo paradigma en ese tipo de estudios, no sólo ante una nueva ampliación de los tipos y modelos de innovación. Para ello, se analizan algunos de los primeros marcos conceptuales de la innovación social (Goldenberg y grupo Crises en Canadá, Young Foundation y NESTA en el Reino Unido) y se comenta el avance del nuevo paradigma en la Unión Europea. Se concluye que las tecnociencias de innovación social parecen haber llegado a un punto de amplio consenso: las innovaciones sociales surgen en primera instancia de la sociedad civil, pero también pueden ser generadas o implementadas por el sector público y por el sector privado. Además, tanto los medios como los fines para promoverlas han de ser predominantemente sociales.

PALABRAS CLAVE: Innovación social; políticas de innovación; tecnociencias sociales.

alcanzable en 2010. La CE diseñó entonces una nueva estrategia para 2020, de la que nos ocuparemos en el apartado 4. Primero queremos señalar algunas insuficiencias en la concepción de innovación entonces usada por la Comisión Europea, dos de las cuales se detectan claramente en la mencionada comunicación de 2003.

1) En primer lugar, la innovación no sólo procede de la investigación científica. El modelo lineal predominó durante años en los diversos países y regiones europeas, de ahí el uso generalizado de las siglas de I+D+i: las actividades e inversiones de cada país en I+D se miden según las normas, metodologías y sistemas de indicadores del Manual de Frascati, mientras que los procesos de innovación son analizados y medidos conforme a las indicaciones del Manual de Oslo, cuya primera edición fue publicada en 1992. Posteriormente, las políticas de innovación de la UE se han inspirado en el modelo sistémico (Nelson 1993, Lundvall 
1993), como se dice explícitamente en la comunicación de 2003: "La evolución del concepto de innovación, que surge de un modelo lineal en el que la política de investigación y desarrollo es el punto de partida hacia un modelo sistémico en el que la innovación nace de interacciones complejas entre los individuos, las organizaciones y sus respectivos entornos operativos, demuestra que las políticas de la innovación no deben concentrarse solamente en la relación entre innovación e investigación"5.

Esta afirmación es importante, por novedosa, pero no implica una pérdida de influencia del modelo lineal. De hecho, en el mismo documento se dice también que: "la explotación de una invención surgida en un laboratorio de investigación es una vía importante, y ampliamente examinada, para la innovación; la investigación aporta una contribución esencial a la innovación, ya que genera un flujo de conceptos tecnológicos y renueva permanentemente la reserva de competencias técnicas" ${ }^{\prime \prime}$. Anthony Arundel y Hugo Hollanders, investigadores del Instituto MERIT (Maastricht Economic Research on Innovation and Tecnology), han llegado a afirmar que el modelo lineal sigue estando plenamente vigente bajo el disfraz de la concepción sistémica de la innovación:

\begin{abstract}
"the countless announcements of the death of the sciencepush or linear model of innovation, based on R\&D, and its presumed replacement with 'systemic' models using Schumpeterian definitions of innovation are decidedly premature. The science-push model based on R\&D is probably the dominant model in use today by both academics and the policy community. Its continued success is partly due to its successful incorporation of many of the features of modern innovation theory. These include shifting final outputs from patents to market indicators and evaluating the effect of a range of business strategies. The disadvantage is that this model largely ignores innovation that is not based on R\&D"7.
\end{abstract}

2) A partir de 2003, la UE amplió su concepto operativo de innovación, ateniéndose a la evolución a nivel internacional (ONU, USA, OCDE, Eurostat). Dicha expansión se plasmó en la tercera edición del Manual de Oslo (2005), donde se distinguen cuatro grandes modalidades de innovación: innovación de productos, de servicios, organizativa y de mercadotecnia. Cada una de ellas fue definida con precisión y empezó a ser estudiada empíricamente mediante los correspondientes sistemas de indicadores ${ }^{8}$. Sin embargo, muy pocos años después se ha comprobado que esta nueva ampliación del concepto de innovación también ha resultado insuficiente. Como alternativa, ha surgido el concepto de innovación social, que analizaremos en el apartado 4. Ese giro social implica un cambio de paradigma, no sólo una expansión o modificación del paradigma anterior. Ésta es la tesis principal del presente artículo. Tanto la tercera edición del Manual de Oslo (2005) como la comunicación de la CE en 2003 aceptaron el paradigma economicista que ha imperado en las politicas y en los estudios de innovación desde los años 80, aunque lo ampliaban a nuevas modalidades de innovación. Dicho paradigma está basado en dos principios:

\section{A) Crear valor consiste en crear valor económico.}

B) Los agentes que desempeñan esa función son las empresas ${ }^{9}$.

La aceptación del paradigma economicista es muy clara en el documento que comentamos, al menos en relación al punto B:

"Los beneficios económicos de una explotación eficaz de la novedad se obtienen gracias a las empresas. La empresa se encuentra en el centro del proceso de innovación. La política de la innovación debe dar finalmente sus frutos, por tanto, en las empresas, mejorando su comportamiento, capacidades y entorno operativo" ${ }^{10}$.

El éxito o el fracaso de las innovaciones tecnológicas se manifiesta en los mercados, por ejemplo al ofertar un producto a un precio más bajo que la competencia. Sin embargo, una empresa puede ser más competitiva si innova y presenta nuevos productos o servicios, que reactivan los mercados. También puede serlo si hace mejor publicidad o comercializa mejor (innovación de marketing), o si promueve un cambio organizativo dentro de la empresa que reduce los costes o incrementa la producción (innovación organizativa). En todo caso, la innovación es un medio para incrementar la productividad y la competitividad de las empresas" 1 . La CE aceptó plenamente ese paradigma dominante: "es la empresa la que organiza la creación de valor"12. Ello no impidió reconocer que la innovación también tiene un componente social. De hecho, la definición de innovación que la CE utilizaba en 2003 era la siguiente: 
"la innovación consiste en producir, asimilar y explotar con éxito la novedad en los ámbitos económico y social" ${ }^{13}$. Se abría así la puerta hacia un giro social en las políticas de innovación, aunque ese umbral no se franqueó entonces. El concepto de innovación social ni siquiera aparecía en documentos oficiales. El paradigma dominante, de origen schumpeteriano, concebía a la sociedad como un ámbito donde las innovaciones se difundían, pero en ningún caso como un agente potencialmente innovador. Innovan las empresas, la sociedad es un ámbito donde hay clientes por captar y fidelizar, en dura competencia con otras empresas. Muchas propuestas innovadoras no tienen éxito en los mercados porque no alcanzan un grado suficiente de difusión y aceptación social. Tanto en el modelo lineal como en la concepción sistémica de la innovación, el contexto social es importante; pero ello no obsta para que esté vigente el principio $B$, según el cual las empresas son el agente innovador por antonomasia, si no el único. La difusión social plantea problemas de comercialización, mercadotecnia y competitividad en los mercados, cuestiones que las empresas han de prever y solucionar si quieren tener éxito con sus innovaciones. Ésta es una de las razones por las que, desde Schumpeter, se distingue la innovación de la invención. La primera es un proceso, tiene un recorrido y pasa por fases. Si la OCDE, el Eurostat y la Comisión Europea ampliaron el concepto de innovación, incluyendo la innovación de marketing como cuarta modalidad, fue precisamente por la importancia que tienen la difusión y la aceptación social de las propuestas innovadoras. Sin embargo, no renunciaron al principio básico: los agentes innovadores son las empresas.

\section{Politicas de innovación en la Agenda DE LISBOA REVISADA}

Al no lograrse los objetivos marcados en la Agenda de Lisboa, la UE remodeló su estrategia en 2005, incluidas sus políticas de innovación ${ }^{14}$, llegando a decir en el informe Aho de 2006 que "all forms of innovation need to be promoted, for innovation comes in many forms others than technological innovation, including organisational innovation and innovation in services"15. Sin embargo, el informe Aho tampoco habla de innovación social, porque permanece en el marco conceptual de la tercera edición del Manual de Oslo. Pese a ello, dicho informe supuso un avance importante, sobre todo al afirmar que había que promover la cultura de la innovación en toda la sociedad y al propugnar que las sociedades europeas fuesen innovation-friendly. Aun así, siguió afirmando que las propuestas innovadoras las hacen las empresas y su éxito o fracaso social se dilucida en los mercados.

En suma, el paradigma anteriormente dominante quedó puesto en cuestión en 2006 en más de un punto, pero no se dio el paso hacia un paradigma alternativo, por mucho que se volviera a hablar de la crisis del modelo lineal y de la necesidad de un paradigma alternativo. Todas esas matizaciones no impidieron que el nuevo Programa Marco 2007-2013 fuese denominado Competitiveness and Innovation Programme (CIP) ${ }^{16}$. El objetivo principal de la Agenda Renovada de Lisboa siguió siendo el del 2000: lograr que la UE fuese económicamente más competitiva que los EE.UU. y Japón, añadiendo ahora algún pais emergente (China). La innovación era un medio para ello. El paradigma economicista siguió estando vigente, aunque ampliado y modificado en puntos importantes.

\section{EMERGENCIA dEL PARADIGMA dE LA INNOVACIÓN social en Canadá y Gran Bretaña}

En contraste con las tendencias dominantes en la OCDE, el Eurostat y la Comisión Europea, en algunos paises se había comenzado a formular un paradigma alternativo desde principios de siglo, tanto fuera de la UE (Canadá, Australia, Nueva Zelanda) como en algún país europeo (Gran Bretaña). En este apartado nos ocuparemos del giro social en los estudios de innovación que tuvo lugar en Canadá y en el Reino Unido, porque grupos de ambos paises han sido los que más han promovido la innovación social como paradigma alternativo a la innovación tecnológica.

En diciembre de 1997, el Gobierno de Quebec diseñó una política de innovación y, entre otras acciones, encargó al Conseil de la science et de la technologie (CST) que elaborara un estudio sobre la contribución que podrían tener las ciencias sociales y las humanidades a una política planificada de innovación. Se creó el Groupe de Travail sur I'Innovation Sociale (GTIS), que redactó un primer documento titulado Recherche en sciences humaines et sociales et innovations sociales, contributions à une politique de 
l'inmatériel (1999). El informe final del CST fue entregado a principios del año 2000 y se tituló Innovation sociale et innovation technologique: l'apport de la recherche en sciences sociales et humaines ${ }^{17}$. Ambos documentos fueron pioneros en los estudios de innovación social y aportaron una propuesta interesante, al Ilamar la atención sobre la existencia de prácticas sociales innovadoras, a las que denominaban innovaciones sociales ${ }^{18}$.

El paradigma emergente todavía resultaba difuso, pero el GTIS apuntó un primer criterio distintivo: las innovaciones sociales afrontan problemas sociales, no sólo empresariales, tecnológicos, de mercado o de producción. Sin embargo, el modelo lineal no era puesto en cuestión, puesto que se daba por supuesto que esas innovaciones sociales provenian de la investigación científica en ciencias sociales, por ejemplo sobre nuevos servicios o nuevas formas de organización.

En noviembre de 2003 se publicó en Canadá un segundo documento que puede ser considerado como un clásico de los estudios de innovación social en el siglo XXI. Fue obra de Julie Cloutier, del grupo Crises (Centre de Recherche sur les innovations sociales, Universidad de Quebec en Montreal), quien realizó un estudio muy detallado de lo que podriamos denominar la "prehistoria de los estudios de innovación social", que ella remite al artículo de James B. Taylor, "Introducing the social innovation" (1970) ${ }^{19}$.

Particularmente incisivo fue el informe Goldenberg, publicado en 2004 por la red canadiense de investigación sobre políticas científicas (CPRN). Ya en el título se apuntaba hacia una nueva concepción de la innovación: Social Innovation in Canada - How the non-profit sector serves Canadians... and how it can serve them better. Goldenberg se interesaba por los procesos de innovación que surgen de la sociedad civil, más concretamente a nivel comunitario. Según él, la innovación social proviene ante todo del nonprofit sector, propuesta clave del paradigma emergente, porque afirma que, aparte de las empresas que buscan beneficios económicos, hay otros agentes innovadores en sectores sociales que no tienen como horizonte los negocios. Esta idea fue afirmada dos años después por la Fundación Young en Gran Bretaña, a través del influyente escrito de G. Mulgan, Social Innovation (2007), donde se distingue claramente la social innovation de la business innovation $^{20}$. Este marco conceptual alternativo también ha sido adoptado por el NESTA británico (National Endowment for Science Technology and Arts), institución que ha publicado numerosos estudios sobre innovación desde 2006, particularmente en los ámbitos de la innovación social, cultural y artística ${ }^{21}$.

El documento de la Young Foundation fue un apoyo teórico importante para la estrategia política del primer ministro británico Tony Blair cuando creó la Cabinet Office of the Third Sector, que a su vez promovió el Social Enterprise Action Plan en 2006, uno de los grandes referentes en las políticas de innovación social, porque aplicó el concepto de empresa social. La política laborista en materia de innovación culminó en 2008, cuando se hizo público el Libro Blanco Nation Innovation, en el que se presentó un plan sistemático para convertir al Reino Unido en un referente mundial en políticas de innovación, incluida la innovación social ${ }^{22}$. Por tanto, el modelo propuesto por Goldenberg tuvo influencia en algunos paises europeos, incluida Suecia, donde la Fundación Vinnova encargó un estudio minucioso de las políticas canadienses de innovación, con la finalidad de tomar ejemplo de ellas ${ }^{23}$. El propio Goldenberg, junto con Wathira Kamoji, Larry Orton y Michael Williamson, ha actualizado recientemente (2009) su informe de 2004, analizando a fondo la evolución del concepto de innovación social en estos cinco años, que ha sido vertiginosa ${ }^{24}$. El Gobierno canadiense no siguió en 2004 los consejos de Goldenberg, pero sí lo ha hecho en 2009, promoviendo por fin políticas de innovación social. Otro tanto ha hecho el Presidente Obama en EE.UU., al crear a finales de 2009 la Office of Social Innovation en la Casa Blanca, la cual gestiona el Social Innovation Fund, dotado con 50 millones de dólares anuales hasta el 2015. Aparte de Gran Bretaña, Canadá y EE.UU., también Australia y Nueva Zelanda han promovido programas políticos para promover la innovación social, no sólo la innovación tecnológica ${ }^{25}$. Por tanto, pensamos que el giro social en las políticas de innovación se produce a partir de 2004, gracias a los informes canadienses (Crises, Goldenberg) y a los subsiguientes estudios británicos (Young Foundation, NESTA). Obvio es decir que estas nuevas políticas de innovación dieron lugar a la creación en estos países de diversos centros de estudios e investigaciones sobre la innovación social ${ }^{26}$.

Este giro social en los estudios de innovación ha quedado bien sintetizado en el segundo informe Goldenberg (2009): 
"the role of the non-profit sector remains critically important, but social innovation is seen today as a broader concept -one that cuts across the non-profit, for-profit and public sectors and that addresses a wider and more complex range of societal issues"27. En su origen, la innovación social surge de la sociedad civil (tercer sector: cooperativismo, movimientos sociales, voluntariado, ecologistas, organizaciones de caridad, etc.), pero progresivamente se incorporan a ella iniciativas procedentes del sector público (programas de asuntos sociales orientados a la innovación, innovación educativa, innovación en los servicios y administraciones públicas, innovación urbana, etc.) y también algunas empresas (empresariado social, filantropía, fundaciones, responsabilidad social corporativa, etc.). Hoy en día se acepta que cualquiera de los tres sectores puede generar innovaciones sociales, en particular cuando colaboran entre sí. Ésta será la segunda propuesta del presente artículo.

\section{EL PARADIGMA EMERGENTE dE INNOVACIÓN SOCIAL EN LA UNIÓN EUROPEA}

¿Cómo ha reaccionado la Unión Europea ante el paradigma emergente? Como vimos, el informe Aho (2006) dejó abierta la vía hacia las políticas de innovación social, pero no las recomendó. Las primeras políticas europeas de innovación social fueron impulsadas por paises concretos, como Gran Bretaña, Finlandia ${ }^{28}$, Suecia ${ }^{29}$ y pocos más, a veces mediante programas diferenciados, en otras ocasiones integrando esas acciones en las políticas generales de innovación. Sobresale el trabajo realizado por el NESTA británico, que ha publicado numerosísimos estudios e informes sobre las diversas modalidades de innovación. A nuestro modo de ver, el NESTA lidera hoy en día los estudios de innovación a nivel europeo, e incluso mundial. Particular interés tienen sus reflexiones sobre las innovaciones ocultas (hidden innovations), concepto muy importante, del que nos ocuparemos más adelante.

La rectificación por parte de la UE de la Agenda de Lisboa 2000, dándole un impulso social y cultural más fuerte, trajo consigo un cierto giro social en los estudios europeos de innovación, pero dicho cambio de dirección apenas si se ha plasmado en las políticas europeas de innovación, salvo en paises concretos. Sin embargo, en 2010 y 2011 se están produciendo cambios significativos en las políticas de innovación de la UE, a la vista de la pujanza que dichas políticas están adquiriendo en países como EE.UU., Canadá y otros ${ }^{30}$. De hecho, el concepto de innovación social se usa cada vez más en la investigación académica, así como en las políticas públicas y en las estrategias empresariales en los países desarrollados. También se usan conceptos similares (responsabilidad social corporativa, marketing social, capital social, economía social, emprendizaje social, etc.), por lo que el concepto de innovación social corre el riesgo de convertirse en una etiqueta carente de rigor y precisión $^{31}$. El Banco Mundial y la OCDE, por ejemplo, han hecho suya la noción de capital social y han definido indicadores para medirlo. Por su parte, la UNESCO impulsa un programa de innovación social en América Latina y el Caribe, con el patrocinio de la Fundación Kellogs. Particular relevancia ha tenido el World Economic Forum Annual Meeting de 2009, organizado por la Schwab Foundation y celebrado en Davos, en el que hubo una sección sobre Social Innovation in a Post-Crisis World ${ }^{32}$.

En este artículo comentaremos únicamente las propuestas del NESTA británico y de la Young Foundation, porque han sido las dos instituciones pioneras de los estudios de innovación en Europa.

El NESTA británico (National Endowment for Science, Technology and Arts) publicó en 2006 y 2007 dos importantes informes sobre, titulados Innovation Gap (octubre de 2006) y Hidden Innovation (junio de 2007). En ambos se planteaba y argumentaba una hipótesis importante: "science-based innovation represents only one important dimension of innovation" ${ }^{33}$. De hecho, el NESTA estimó que sólo el 6\% de la innovación en la economía británica estaba basada directamente en la investigación científica y que buena parte de los procesos de innovación no eran medidos por los sistemas de indicadores usuales $(O C D E$, Eurostat, Manual de Oslo, Innometrics, etc.). Surgió así la hipótesis de la innovación oculta (hidden innovation), que el NESTA definió como: "the innovation activities that are not reflected in traditional indicators such as investments in formal R\&D or patents awarded; despite not being measured, hidden innovation often represents the innovation that matters - the innovation that most directly contributes to real practice and performance of a sector ${ }^{134}$.

El Libro Blanco Nation Innovation que publicó el Gobierno británico en 2008 hizo suyas las principales hipótesis 
del NESTA y comenzó a concebir la innovación como un proceso multidimensional, que en ocasiones surge de los departamentos de I+D de las empresas, pero en la mayoría de las ocasiones tiene orígenes ajenos a la I+D. Dicho en términos kuhnianos, estamos ante una importante anomalía del paradigma vigente, que pone en cuestión su núcleo duro, la I+D+i, puesto que buena parte de los procesos de innovación, posiblemente más de la mitad, quedarían fuera de los sistemas de medición utilizados. Ésta es una de las razones por las que Pro-Innova ha modificado recientemente sus sistemas de indicadores (European Innovation Scoreboard, EIS, Regional Innovation Scoreboard, RIS) y ha creado el Union Innovation Scoreboard (UIS), en el que se introducen indicadores de innovación para los procesos de innovación no basados en la I+D. Todo ello refuerza la tendencia hacia un cambio de paradigma en los estudios europeos de innovación. Visto desde otra perspectiva, pudiera ocurrir que los instrumentos para medir la innovación no sólo sean insuficientes, sino que constituyan una parte importante del problema. Así lo sugirió el NESTA en su documento Hidden Innovation: "historically, the development of metrics has proven a substantial barrier to the extension of innovation policy beyond science and technology"35. Puesto que las políticas de I+D priman la I+D, financian ante todo ese tipo de actividades de innovación, dejando de lado las restantes, que son muchas y diversas. De ser esto cierto, el cambio de paradigma en los estudios de innovación no se limitaría a un debate académico, sino que implicaría la elaboración de nuevos sistemas de indi-

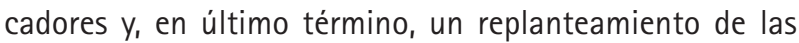
políticas de innovación, que históricamente han surgido de la $I+D$, pero que deberian superar esa limitación originaria ${ }^{36}$. No olvidemos el carácter proactivo de los sistemas de indicadores, que fue subrayado en uno de los informes metodológicos de la Trend Chart europea (2006):

\begin{abstract}
"the Trend Chart project is proactive - the thematic reports and the EIS (European Innovation Scoreboard) are designed to provide indicators and analysis that are relevant to current policy concerns. For example, the selection of EIS indicators for the drivers and characteristics of innovation is partly determined by the policy relevance of these indicators" ${ }^{137}$.
\end{abstract}

Dicho en otros términos: los estudios de innovación no son científicos, sino tecnocientíficos. No pretenden conocer lo que pudieran ser los procesos de innovación en toda su amplitud y diversidad, sino generar terminologías, métodos e indicadores que permitan medir y comparar los procesos de innovación entre sí, y todo ello al servicio de estrategias empresariales o de políticas públicas de fomento de la innovación, vinculadas a unos intereses y valores. Ello equivale a decir que no estamos ante paradigmas en el sentido de Kuhn, sino ante tecnoparadigmas, es decir, ante un conocimiento proactivo que no versa sobre el know why sino sobre el know how y el how doing. Las tecnociencias se diferencian de las ciencias por estar orientadas a la transformación del mundo, más que a conocer cómo es. En el caso de las tecnociencias sociales, se trata de transformar las sociedades y orientarlas en una determinada dirección, convirtiéndolas en sociedades de la información, o del conocimiento, como pretendió la Agenda de Lisboa 2000. Desde el informe Aho, en cambio, se ha empezado a hablar de una Europa innovadora y de sociedades innovadoras, e incluso de naciones innovadoras ${ }^{38}$. Los estudios de innovación son una modalidad de tecnociencia social y por eso son proactivos. Los sistemas de indicadores son herramientas que sirven para implementar determinadas políticas activas, normalmente con el fin de crear o de transformar los sistemas nacionales, regionales o locales de innovación. Los tecnoparadigmas (Manual de Oslo, European Innovation Scoreboard, etc.) son ante todo sistemas de know how que determinan cómo hay que conceptualizar, definir y medir la innovación que interesa medir y se definen en función de dichas políticas, aunque mucha innovación quede fuera de la métrica vigente, como señaló el NESTA al hablar de la hidden innovation. Por nuestra parte, prevemos que estos cambios conceptuales y metodológicos en los estudios de innovación son el preludio de cambios en las correspondientes políticas, por eso intentamos anticiparnos los nuevos marcos conceptuales.

Pues bien, los estudios británicos sobre innovación social acabaron influyendo en Bruselas. De hecho, en diciembre de 2008 el Consejo de Europa convocó diversos paneles de expertos, con el fin de elaborar un Plan Europeo sobre Innovación que modificaría los anteriores. Para dar los primeros pasos en esa dirección, el BEPA (Bureau of European Policy Advisers) organizó los días 19 y 20 de enero de 2009 un taller en Bruselas sobre innovación social: "the objective was to debate how Europe supports and integrates social innovation in its policies, in the wake of its renewed agenda" ${ }^{\prime 3}$. Participaron en los debates 40 expertos en innovación social, entre ellos el Director de la Young 
Foundation, Geoff Mulgan, así como su Director Asociado, Rushanara Alí. También asistieron dos Comisarios de la UE, el vicepresidente del European Investment Bank y el propio Presidente de la Comisión Europea, José Manuel Barroso, quien hizo declaraciones en pro de la innovación social, demostrando la importancia que la Comisión pensaba atribuir a la nueva línea de trabajo. Las actividades en esta dirección continuaron a lo largo del año $2009^{40} \mathrm{y}$ en enero de 2010 el BEPA encargó oficialmente a la Young Foundation y a la red mundial de Innovación Social, SIX, que elaboraran un estudio sobre la innovación social en Europa. A diferencia de los estudios iniciales de la Young Foundation, que fueron de índole más académica, aunque influyeron en las políticas británicas de innovación, el nuevo documento, Study on Social Innovation (2010), fue elaborado expresamente para orientar el diseño de las políticas de la UE. Dicho estudio constituye un punto clave para el giro social de las políticas europeas de innovación y por ello terminaremos este artículo comentándolo brevemente ${ }^{41}$.

\section{El Study on Social Innovation de la Young Foundation y La RED $S I X$}

La Young Foundation británica ha desempeñado un papel muy importante en los estudios de innovación social, en particular desde la publicación del influyente escrito de Geoof Mulgan, Social Innovation: what is it, why it matters, how it can be accelerated (2007). Dicho informe tuvo continuidad en estudios ulteriores, redactados a veces en colaboración con el NESTA. También fue importante la iniciativa de crear la red mundial SII ${ }^{42}$, que aglutina la información sobre la innovación social en todo el mundo.

En el documento de 2007, Mulgan mencionaba numerosos ejemplos históricos de iniciativas que, a su juicio, fueron innovaciones sociales relevantes: aparición de los sindicatos, cooperativismo -mencionando el de Mondragón-, jardines de infancia, equipos deportivos, clubes de lectura, organizaciones financieras de microcréditos, organizaciones filantrópicas y de caridad, etc. También aludía a diversos autores que habian escrito sobre el tema (Everett Rogers, Christopher Freeman, Rosabeth Moss Kanter, William Baumol, Eric Von Hippel, Bart Nooteboom, Clay Christianson y John $\mathrm{Kao}^{43}$ y y distinguía diversos ámbitos donde pueden producirse hoy en día procesos de innovación social, por ejemplo el emprendizaje social, el diseño, la tecnología, las políticas públicas, las ciudades (innovación urbana), los movimientos sociales y el desarrollo comunitario ${ }^{44}$. Como ejemplos recientes de innovación social la Young Foundation mencionaba los siguientes:

\begin{abstract}
"Self-help health groups and self-build housing; telephone help lines and telethon fundraising; neighbourhood nurseries and neighbourhood wardens; Wikipedia and the Open University; complementary medicine, holistic health and hospices; microcredit and consumer cooperatives; charity shops and the fair trade movement; zero carbon housing schemes and community wind farms; restorative justice and community courts. All are examples of social innovation new ideas that work to meet pressing unmet needs and improve peoples' lives" 45 .
\end{abstract}

La última frase resume la definición general de innovación social que Mulgan y sus colaboradores usaron a partir de 2006, aunque en el mismo documento también propusieron otra definición, todavía más precisa:

"innovative activities and services that are motivated by the goal of meeting a social need and that are predominantly developed and diffused through organisations whose primary purposes are social"46.

Por tanto, la Young Foundation no sólo caracterizaba la innovación social por sus objetivos (satisfacer o resolver problemas o necesidades sociales), sino que exigía además que las iniciativas socialmente innovadoras hubieran sido impulsadas, al menos inicialmente, por agentes cuyos objetivos fuesen primordialmente sociales, no políticos, económicos ni empresariales. Se introducía así una definición más restringida de innovación social, si la comparamos con la propuesta de Goldenberg en Canadá, aunque emparentada con ella.

Algunos documentos recientes de la Fundación, elaborados en colaboración con la red SIX (Social Innovation eXchange) han matizado las posturas iniciales de Mulgan y sus colaboradores, en particular el Study on Social Innovation de 2010. Al haber sido redactado a petición del BEPA y de la Comisión Europea ${ }^{47}$, en este estudio se flexibiliza el marco conceptual y se tiende a una sintesis pragmática con otras concepciones de la innovación social. El cambio más 
significativo consiste en reconocer que, además del tercer sector, la innovación social también puede provenir de iniciativas del sector privado y del sector público, siempre que aborden necesidades sociales de forma novedosa o afronten problemas sociales significativos. Esta idea ya aparecía en las publicaciones anteriores de la Fundación, pero ahora queda mucho más afirmada desde el principio:

"It (the Report) looks at innovations in all sectors that are achieving high impact and productivity, particularly in relation to goals of better health, education, employment or the environment, rather than focusing exclusively on the public, private or non-profit sectors" 48 .

Por tanto, hay un cambio metodológico y conceptual interesante. En lugar de distinguir los tipos de innovación social por los agentes que la promueven, como había sido habitual en los escritos previos de la Young Foundation, el Study se adecua en parte a los objetivos de la Comisión Europea y se centra en los sectores donde la innovación social puede tener mayor impacto social y ser más productiva: salud, educación, empleo y medio ambiente. Al proceder así, la Young Foundation evolucionó de manera similar a como lo hizo Goldenberg y su grupo canadiense: adecuando el marco conceptual propuesto a las prioridades políticas. A cambio, se afirmó taxativamente que la innovación social debería estar en el centro de la estrategia de la Unión Europea cara al 2020, por lo que a las políticas de innovación respecta:

\footnotetext{
"Just as the Lisbon Strategy for jobs and growth focused on innovation, entrepreneurship and the knowledge-based economy, the new strategy for Europe, Europe 2020, must have social innovation at its centre, as a means of stimulating a more dynamic, inclusive and sustainable social market economy"49.
}

¿Supone esto el anuncio de un giro social en las políticas europeas de innovación? Por el momento, la afirmación de que la innovación social ha de estar en el centro de la estrategia europea se hace desde el ámbito de los estudios de innovación. Falta por ver si este tipo de propuestas serán recogidas o no por los responsables en la elección de las estrategias de la UE y de sus ejes principales. En cualquier caso, queda claro que los estudios de innovación social se presentan como una auténtica alternativa al paradigma dominante. En la Agenda de Lisboa del año 2000, la innovación social ni siquiera era mencionada, sólo se hablaba de la innovación tecnológica y empresarial. Otro tanto ocurrió en 2003, cuando la Comisión Europea definió su política de innovación. En cambio, en el año 2010 la innovación social está siendo propuesta como un nuevo eje para las políticas europeas ${ }^{50}$. De hecho, la reciente Europe Flagship 2020 Initiative, denominada Innovation Union, al modo del Gobierno británico en 2008 con su Innovation Nation, establece diez grandes líneas de acción para conseguir que la Unión Europea sea altamente innovadora en 2020 y en la novena de ellas se afirma taxativamente: "we must champion in social innovation"51. Como primer paso en esa dirección, la Comisión Europea convocó en febrero de 2010 la European Initiative for Social Innovation, con el fin de destacar algunas buenas prácticas $y$, de paso, clarificar la noción de innovación social mediante un conjunto de ejemplos paradigmáticos. Sin embargo, faltan otras acciones para poner en marcha un programa de fomento de la innovación social, que se concretarán en el próximo Programa Marco, previsiblemente. De lo contrario, la Unión Europea no será el líder mundial en 2020, puesto que ya hemos subrayado que otros paises también han empezado a desarrollar políticas de fomento de la innovación social.

Al proponer la innovación social como línea estratégica alternativa, la Young Foundation y el NESTA fueron conceptualmente prudentes, quizá por eso sus propuestas han tenido inicialmente éxito. Ambas instituciones han criticado repetidas veces que las políticas de innovación de la UE hubieran estado basadas únicamente en la $1+D^{52} y$ han hecho propuestas alternativas, como la innovación de usuarios $^{53}$. De hecho, el NESTA ha publicado recientemente un estudio muy interesante, Measuring User Innovation in the $U K^{54}$, en el que insiste en que los usuarios son una fuente importante de innovación, más eficiente que los departamentos de I+D. En suma, la red SIX y la Young Foundation han optado en su Study por una actitud pragmática. Afirman que la innovación social no es lo mismo que empresa social (social enterprise) ni emprendizaje social (social entrepreneurship), pero también admiten que dichos términos se solapan entre si ${ }^{55}$. Por tanto, hay formas mixtas de innovación, innovaciones socio-económicas y tecnosociales, por asi llamarlas. Por nuestra parte, nos parece una opción adecuada en el momento actual. El giro social en los estudios de innovación no está lo suficientemente consolidado como para poder apoyar eficazmente 
las nuevas políticas de innovación de la UE: aunque se dispone de un marco conceptual como el que ha sido presentado en el Study o en el ulterior Open Book on Social Innovation, faltan sistemas de indicadores para comparar las iniciativas y proyectos socialmente innovadores. Por otra parte, la pluralidad de agentes innovadores posibles ha de traslucirse en bases de datos donde esos agentes estén identificados. Mientras los policy makers no dispongan de estos y otros instrumentos tecnocientíficos, la sustitución de un paradigma por otro no se llevará a cabo. Podemos concluir que la innovación social conforma un paradigma alternativo, pero no llega a ser un tecnoparadigma, porque carece de unas herramientas operativas que sirvan de ayuda para orientar las políticas científicas. Construir sistemas de indicadores de innovación social es una tarea urgente en este ámbito de estudios, y a ello se está dedicando nuestro grupo de investigación en la Universidad del País Vasco.

Como conclusión final, de índole más conceptual, diremos que la Young Foundation y el grupo canadiense de Golbenberg coinciden en un punto clave, referido a las fuentes de la innovación social:

"Social innovation describes the process of invention, diffusion and adoption of new services or organisational models, whether in the non-profit, public or private sector" ${ }^{156}$.

Éste es el marco en el que nuestro grupo investiga en el Laboratorio de Innovación Social de la Universidad del País Vasco. Aceptar este criterio implica investigar tres tipos de procesos de innovación social, según procedan de uno de los tres sectores. Según la Young Foundation, tanto el Stanford Social Innovation Report norteamericano como el NESTA británico, incluso el Forum on Social Innovations de la OCDE, podrían coincidir en este punto, razón por la que podemos afirmar que la evolución de los estudios de innovación social en la primera década del siglo XXI parece haber llegado a un punto de amplio consenso, lo que no es poco: las innovaciones sociales surgen, en primera instancia, de la sociedad civil, pero también pueden ser generadas o implementadas por el sector público y por el sector privado. Todo depende de los fines y los medios. La nueva definición de innovación social que proponen la red SIX y la Young Foundation en el Study de 2010 que estamos comentando es la siguiente:

"Social innovations are innovations that are social both in their ends and in their means"57.

Si a ello le añadimos que los agentes que promueven las innovaciones sociales pueden provenir de los tres sectores ya mencionados, quedaría suficientemente definido el marco conceptual en el que se mueve el paradigma alternativo que hemos comentado en este artículo.

Por lo que a nosotros respecta, nos parece una solución de compromiso, aunque tiene algunos defectos, sobre todo su apelación a la racionalidad instrumental de medios y fines. Filosóficamente hablando, sería preferible hablar de valores, en lugar de medios y fines, puesto que tanto los medios elegidos como los objetivos de las propuestas innovadoras han de ser valorados, positiva o negativamente. En este sentido, cabría distinguir entre innovaciones económicas, sociales, tecnológicas, empresariales, políticas, jurídicas, administrativas, ecológicas o de otros tipos en función de los diversos tipos de valores que guían las acciones innovadoras y los correspondientes procesos de difusión y aceptación. Sin embargo, a la hora de orientar las políticas científicas el binomio medios/fines está ampliamente aceptado, razón por la cual la definición de consenso que ha propuesto la Young Foundation en 2010 nos parece un buen punto de partida para hacer avanzar los estudios de innovación social. 
1 Este artículo ha sido elaborado en el marco del proyecto de investigación FFI2008-03599/FISO, financiado por el Ministerio de Educación y Ciencia y desarrollado en el Centro de Ciencias Humanas y Sociales del CSIC.

2 La Comisión había publicado previamente dos documentos sobre políticas de innovación, relacionadas con la agenda de Lisboa: la comunicación titulada "La innovación en una economía del conocimiento" (COM 2000, 567) y el informe "La política de innovación en Europa 2002" (Expedientes de la innovación n. $\left.{ }^{\circ} 29,2003\right)$.

3 "Política de la innovación: actualizar el enfoque de la Unión en el contexto de la estrategia de Lisboa", COM (2003) 112 final, p. 5.

4 "Productividad: clave de la competitividad de las economias y empresas europeas", COM (2002) 262.

5 COM (2003) 112 final, p. 8

6 lbid., p. 16.

7 "Missing" indicators of innovation, July 1, 2006, p. 3.

8 Ver Manual de Oslo, capítulo 3, § 3-4.

9 El Manual de Oslo acepta a nivel teórico la existencia de varias modalidades de innovación, pero sólo se ocupa de la empresarial: "La innovación puede estar presente en cualquier sector de la economía, incluyendo los servicios públicos tales como la salud y la educación. Las directrices de este Manual, sin embargo, se han diseñado esencialmente para tratar solamente las innovaciones en el sector empresarial" (§ 27, p. 25).

10 COM (2003) 112 final, p. 8.

11 Manual de Oslo, tercera edición, $2005, \S 1$, p. 3.

Recibido: 8 de junio de 2010

12 COM (2003) 112 final, p. 9.

Aceptado: 18 de octubre de 2010

14 La nueva Agenda Social de la UE para el período 2006-2010 fue definida en COM (2005) 33 final. En cuanto a las políticas remodeladas de innovación, el documento más significativo fue la Comunicación "More Research and Innovation - Investment for growth and employment", COM (2005), 488. En ella se definieron 19 áreas prioritarias y se promovieron acciones estratégicas como la creación de PRO INNO y Europe INNOVA. En aquel momento, ambas agencias estuvieron focalizadas hacia la entrepreneurial innovation. Otro documento básico es "Putting knowledge into practice: A broad.based innovation strategy for the EU", COM (2006), 502 final, en donde se ponen en práctica las recomendaciones del informe Aho.

15 lbid., p. 4.

16 Fue publicado en el Official Journal of the European Union el 9 de noviembre de 2006.

17 Conseil de la Science et la Technologie. Groupe de Travail sur la Innovation Sociale, Recherche en sciences humaines et sociales et innovations sociales, contributions à une politique de l'immatériel. Gouvernement du Québec. Québec, 1999.

18 Conseil de la Science et la Technologie. Groupe de Travail sur la Innovation Sociale, Innovation Sociale et Innovation Technologique: I'apport de la recherche en sciences sociales et humaines. Gouvernement du Québec. Québec, 2000.

19 J. B. Taylor, "Introducing social innovation", Journal of Applied Behavioral Science, 6:1, pp. 69-77. El grupo Crises publicó asimismo en noviembre de 2003 otro documento importante, escrito por Martin Petitclerc y titulado Rapport sur les innovations sociales et les transformations sociales (Cahiers du Crises, Collection Wor- 
king Papers ET0313, Quebec 2003). Petitclerc relacionó los estudios de innovación social con las teorías sociológicas sobre el cambio social.

20 G. Mulgan, Social innovation, what it is, why it matters and how it can be accelerated, London, Young Foundation, 2006, p. 8. El documento fue presentado en 2006 en Beijing y publicado a principios de 2007. En él se aportan dos definiciones de innovación social, una más general ("social innovation refers to new ideas that work in meeting social goals", p. 8) y otra más específica: "innovative activities and services that are motivated by the goal of meeting a social need and that are predominantly developed and diffused through organizations whose primary purposes are social" (Ibid.). Por tanto, una innovación es social, en primer lugar, si sus objetivos son sociales $y_{1}$ en segundo lugar, si los agentes que la promueven son organizaciones con metas sociales. La denominaremos concepción restringida de la innovación social, aunque Mulgan y sus colaboradores de la Young Foundation afirman desde el principio que "social innovation is not unique to the non-profit sector" (/bid., p. 4). Consulta realizada el 2 de marzo de 2008.

21 Verwww.nesta.org.uk/informing/glossaryl. Asumiendo las tesis de Mulgan, el NESTA definía en su glosario-web la innovación social de la siguiente manera: "Activities and services that are motivated by the goal of meeting a social need, diffused through organisations, individuals and enterprises whose primary purposes are social and where profit is re-invested. This is distinguished from innovation in business, which is generally motivated by profit maximisation and so diffused by organisations that seek profit as their main purpose". Consulta realizada el 3 de abril de 2008.

22 Nation Innovation 2008.

23 Ver Thomas Liljemark, Innovation Policy in Canada: Strategy and Realities, Östersund, ITPS, Swedish Institute for Growth Policy Studies, 2005. En cuanto a las políticas de innovación en Suecia, de las que no nos ocupamos en este artículo, el documento básico es Innovative Sweden: A Strategy for Growth through renewal, Ministry of Industry, Employment and Communications, Stockholm, October 2004. No se alude a la innovación social, pero si hay un apartado titulado "Innovative people" (pp. 40-44) orientado hacia la promoción del emprendizaje, que ha estado a cargo de la agencia gubernamental NUTEK.

24 M. Goldenberg, W. Kamoji, L. Orton and M. Williamson, Social Innovation in Canada: An Update, CPRN Research Report, September 2009.

25 Australia creó en 2008 el Australian Center for Social Innovation (ACSI). Nueva Zelanda también creó un Centre for Social Innovation y un New Zealand Social Innovation Investment Group.

26 Aparte de Crises en Quebec y de los centros británicos ya citados (Young Foundation, NESTA), conviene mencionar los centros de Toronto, Stanford, Australia, Nueva Zelanda, Helsinki, Estocolmo y Viena.

27 Goldenberg 2009, p. V.

28 Ilkka Taipale ha publicado un libro en finés titulado "100 innovaciones sociales en Finlandia", que no hemos utilizado, por estar traducido sólo al japonés.

29 La Agencia sueca de innovación, VINNOVA, financia el programa Designing Social Innovation, que ha empezado en abril de 2010 y durará tres años. Está dirigido por Ramia Mazé.
30 A principios de 2009, el Presidente de la Comisión Europea, José Manuel Barroso, anunció la creación de un grupo de expertos sobre innovación social, con el fin de definir la postura de la UE en el Foro Económico Mundial de Davos, donde el tema de la innovación social había sido incluido. Comentaremos el documento de dichos expertos en el apartado final.

31 Así lo afirma Ander Gurrutxaga en Gurrutxaga y Echeverría 2010.

32 Ver Innovations, MIT Press Journal, Davos-Klosters 2009, 216 pp.

33 NESTA 2007, p. 4.

34 NESTA 2007, p. 4.

35 NESTA 2006, p. 5.

36 Lamentablemente, esta tendencia no se atisba en España, a pesar de que acaba de aprobarse en 2011 una Estrategia Estatal de Innovación. Un primer vistazo al documento publicado por el Ministerio de Ciencia e Innovación (http://www.micinn.es/stfls/ MICINN/Innovacion) parece mostrar que dicha estrategia permanece anclada en el anterior paradigma. De hecho, su marco conceptual de partida refleja las posturas estrictamente economicistas del World Economic Forum (Informe de Competitividad Global 2009-2010) y del documento de la OCDE, The OECD Innovation Strategy de mayo de 2010. Por falta de espacio, nos ocuparemos de estos tres documentos en un artículo ulterior, centrado en España.

372006 Trend Chart Methodology Report: Searching the forest for the trees: "Missing" indicators of innovation", Anthony Arundel and Hugo Hollanders, MERIT - Maastricht Economic Research on Innovation and Tecnology, July 1, 2006, p. 3.

38 Es el caso del Innovation Nation británico, antes mencionado, o de la creación de Innobasque en Euskadi. 
39 European Commission, Bureau of European Policy Advisers, Bruselas, D(2009), p. 2.

40 Simposio de junio sobre financiación de la innovación social, coordinado por Diogo Vasconcelos, de la red SIX. Dicho Simposio dio lugar, a propuesta de Mulgan, a la iniciativa This is European Social Innovation, cuyo objetivo consiste en seleccionar las 10 iniciativas actuales de innovación social más relevantes en Europa. Esta iniciativa está impulsada por la Comisión Europea, la red SIX y Euclid Network, una de las organizaciones europeas más importantes en relación a los estudios sobre el tercer sector.

41 Por falta de espacio, en este artículo tampoco nos ocuparemos de un documento posterior de Murray, Caulier-Grice y Mulgan, The Open Book for Social Innovation (marzo de 2010), pese a su indudable interés. Los estudios de innovación social están en plena efervescencia en Europa y aquí sólo nos ocupamos de aquellos estudios que han tenido una influencia directa en las políticas europeas de innovación. Ese libro abierto ha sido publicado conjuntamente por la Young Foundation y el NESTA, institución esta última que ha pasado a ser dirigida por Geoff Mulgan. Está disponible en www.nesta.org.uk/publications.

42 Ver www.socialinnovationexchange. org.

43 Conviene resaltar que no mencionan a Goldenberg ni los estudios canadienses sobre innovación social.

44 Mulgan 2007, p. 6.

45 lbid., p. 7.

46 Ibid., p. 8. Esta definición apenas varía en dos documentos ulteriores de la Young Foundation: In and out of sync, Growing Social Innovations, redactado por $\mathrm{G}$. Mulgan junto con $\mathrm{R}$.
Ali, R. Halkett y B. Sanders (London, Young Foundation, september 2007) y G. Mulgan, Ready or Not? Taking innovation in the public sector seriously (London, NESTA, April 2007). En este último estudio, Mulgan aporta una definición interesante de la innovación en el sector público: "The simplest definition is that public sector innovation is about new ideas that work at creating public value. The ideas have to be at least in part new (rather than improvements); they have to be taken up (rather than just being good ideas); and they have to be useful. By this definition, innovation overlaps with, but is different from, creativity and Entrepreneurship (/bid., p. 6). Por nuestra parte, pensamos que en los procesos de innovación lo importante es crear valor, pero dicho valor puede ser social, político, económico, ecológico, urbano o de otro tipo. Las diversas modalidades de innovación surgen en función del tipo de valores que se crean.

47 Los autores del informe son Julie Caulier-Grice, Lauren Kahn y Geoff Mulgan, por la Young Foundation, junto con Louise Puldorf y Diogo Vasconcelos, por la red SIX.

48 SIX and Young Foundation, Study on Social Innovation, European Union/ Young Foundation 2010, p. 5.

49 lbid.

50 Hemos centrado nuestro análisis en la UE y sus políticas de innovación, pero también podriamos haber aludido a los cambios en la concepción de la innovación que se han producido en la OCDE y que, por el momento, se han plasmado en el documento ya mencionado, OCDE 2010, que no vamos a comentar en este artículo.

51 COM (2010), 546 final, p. 3.
52 SIX and Young Foundation 2010, o.c., p. 13.

53 lbid.

54 Obra de Stephen Flowers y otros autores (2010), entre los cuales está Eric von Hippel, quien defiende la importancia de la innovación de usuarios desde hace un cuarto de siglo.

55 lbid., p. 15.

56 lbid., p. 16.

57 lbid., pp. 17-18.

\section{BIBLIOGRAFÍA}

Bakhski, H.; Schneider, Ph. and Walker, Christopher (2008): Arts and Humanities Research and Innovation, NESTA, Arts and Humanities Research Council, noviembre 2008.

Chesbrough, H. (2003): Open Innovation, Mass., Harvard Business School Press.

Chesbrough, H. (2006): Open Business Models, Mass., Harvard Business School Press.

Cloutier, Julie (2003): Qu'est-ce que I'innovation sociale?, Quebec, Cahiers de Crise.

Deroïan, F. (2002): "Formation of social networks and diffusion of innovations", Research Policy, 31 (5), 835-846.

DIUS (13 marzo 2008): Innovation Nation, Department for Innovation, Universities \& Skills (DIUS), UK Government, Crown.

Echeverría, J. (2008): "El manual de Oslo y la innovación social", Arbor, 732, pp. 609-618.

Echeverría, J. (2008): "Aportaciones preliminares a los estudios de innovación social", en I. Mendiola (ed.), Textos y Pretextos para repensar lo social, Bilbao, Universidad del País Vasco, pp. 81-96.

Echeverría, J. (2010): "De las políticas de investigación a las políticas de inno- 
vación", Acta Sociologica (México), 55:1, pp. 13-37.

Flowers, S.; Von Hippel, E.; De Jong, J. y Sinozic, T. (2010): Measuring User Innovation in the UK, London, NESTA.

Gurrutxaga, A. (2009): Recorridos por el cambio, la innovación y la incertidumbre, Bilbao, Universidad del País Vasco.

Gurrutxaga, A. y Echeverría, J. (2011): La luz de la luciérnaga. Diálogos de innovación social, Madrid, Ed. Plaza y Valdés. Innovation sociale et innovation technologique (2000), Quebec, Conseil de la Science et de la Technologie.

Klein, J. L. and Harrison, D. (eds.) (2006): L'innovation sociale, Quebec, Presses de I'Université de Quebec.

Lundvall, B. A. (1992): National systems of Innovation: Towards a theory of interactive learning, London, Pinter.

Lundvall, B. A. (1998): Innovations as an Interactive Process: From user-producer Interaction to the National System of Innovation, en G. Dosi, C. Freeman, R.
Nelson, G. Silverberg and L. Soete (Eds.), Technical Change and Economy Theory, London, Pinter Publisher, pp. 349-369.

Miles, I. and Green, L. (2008): Hidden innovation in the creative industries, NESTA Research Report, julio.

Mulgan, G. (2007): Social Innovation: what is it, why it matters, how it can be accelerated, London, Young Foundation, Basingstoke Press.

Mulgan, G. et al. (2007): In and out of sync: the challenge of growing social innovations, London, NESTA Report.

Mulgan, G. (2007): Ready or Not: Taking innovation in the Public Sector Seriously, London, Young Foundation.

Murray, R.; Caulier-Grice, J. and Mulgan, G. (2010): The Open Book of Social Innovation, London, Young Foundation and NESTA.

Nelson, R. R. (1993): National Systems of Innovation, Oxford, Oxford Univ. Press.

NESTA (2006): The Innovation Gap, London, NESTA.
NESTA (2007): Hidden Innovation, London, NESTA.

NESTA (2009): The Innovation Index. Measuring the UK's investment in innovation and its effects, NESTA Index Report, noviembre.

Oakley, K.; Sperry, B. and Pratt, A. y H. Bakhski (ed.) (2008): The art of innovation. How fine arts graduates contribute to innovation, NESTA Research Report 16, septiembre.

OECD/European Communities (2005): Os/o Manual: Guideliness forCollecting and Interpreting Innovation Data, 3. ${ }^{a}$ ed., Paris, OECD/EC.

OECD (2010): The OECD Innovation Strategy, Getting a Head Start on Tomorrow, Paris, OECD.

SIX and Young Foundation: Study on Social Innovation, European Union/Young Foundation 2010.

Von Hippel, E. (1988): The sources of innovation, New York, Oxford Univ. Press.

Von Hippel, E. (2005): Democratizing Innovation, Cambridge, MA: MIT Press. 